\title{
Strongly Coupled Quantum and Classical Systems and Zeno's Effect
}

\author{
Ph. Blanchard \\ Faculty of Physics and BiBoS, University of Bielefeld \\ D 33615 Bielefeld, FRG \\ A. Jadczyk \\ Institute of Theoretical Physics, University of Wrocław, \\ Pl. Maxa Borna 9, PL 50204 Wrocław, Poland
}

August 24, 2021

\begin{abstract}
A model interaction between a two-state quantum system and a classical switching device is analysed and shown to lead to the quantum Zeno effect for large values of the coupling costant $\kappa$. A minimal piecewise deterministic random process compatible with the Liouville equation is described, and it is shown that $\kappa^{-1}$ can be interpreted as the jump frequency of the classical device.
\end{abstract}

\section{Introduction}

Generations of freshmen in philosophy have discussed the memorable puzzles of Zeno. The most important are about motion, 'Achilles' and the 'flying arrow' being the leading ones:

(i) Achilles cannot overtake a turtle, since whenever he reaches the position from which the turtle started the turtle has by then a new lead.

(ii) If time is composed of moments, a would-be flying arrow must at each moment be at rest, and thus can never fly.

For quantum systems repeated frequent measurements of survivals can prevent the decay of the quantum state. This effect was formulated by Turing 1940 and called 
1977 quantum Zeno effect by B. Misra and E.C.G. Sudarshan [1]. In other words: repeated measurements keep the state from evolving. In recent years there has been considerable discussion of the quantum Zeno processes, effect and paradox. See for example [2, 3, [, 5]. Moreover it has been claimed that experiments can demonstrate the effect [6, 0, 8]. In a recent paper [9] we propose a mathematically consistent model of interaction between classical and quantum systems, which provides an answer to the question of how and when a quantum phenomenon becomes real as a result of a suitable dissipative time evolution. With a properly chosen initial state the quantum probabilities are exactly mirrored by the state of the classical system and moreover the state of the quantum subsystem converges for $t \rightarrow+\infty$ to a limit which agrees with that required by von Neumann - Lüders, standard quantum measurement projection postulate. In our model the quantum system is continuously coupled to a classical apparatus which will respond to its time evolution and gives therefore a minimal mathematical semantics to describe the measurement process in Quantum Mechanics. In order to arrive at Zeno's paradox one tacitly involves Schrödinger's equation. But on the other hand we know it holds only for an undisturbed system, whereas in fact we disturb the system very often - say, continuously - by observations. In Section 2 we will briefly describe the model and discuss Zeno's effect in this framework. In the strong coupling limit we will estimate the distance travelled by the quantum state $d(\hat{\rho}(0), \hat{\rho}(t)), d$ being the Bures distance in the state space. Moreover we give a stochastic description for the Zeno's model using piecewise deterministic processes. Section 3 deals with some concluding remarks.

\section{The Zeno's effect revisited}

Let us first very briefly describe the mathematical framework we will use. For details we refer to [9]. We consider a quantum system $\Sigma_{q}$ in interaction with a classical system $\Sigma_{c}$. To the quantum system there corresponds a Hilbert space $\mathcal{H}_{q}$. In $\mathcal{H}_{q}$ we consider a family of orthonormal projectors $e_{i}=e_{i}^{\star}=e_{i}^{2},(i=1, \ldots, n), \sum_{i=1}^{n} e_{i}=$ 1 , associated to an observable $A=\sum_{i=1}^{n} \lambda_{i} e_{i}$. The classical system is supposed to have $m$ distinct pure states, and it is convenient to take $m \geq n$. The algebra $\mathcal{A}_{c}$ of classical observables is in this case $\mathcal{A}_{c}=\mathbb{C}^{m}$. The set of classical states coincides with the space of probability measures. Using the notation $X_{c}=\left\{s_{0}, \ldots, s_{m-1}\right)$, a classical state is am $m$-tuple $p=\left(p_{0}, \ldots, p_{m-1}\right), p_{\alpha} \geq 0, \sum_{\alpha=0}^{m-1} p_{\alpha}=1$. The state $s_{0}$ plays in some cases a distinguished role and can be viewed as the neutral initial state of a counter. The algebra of observables of the total system $\mathcal{A}_{t o t}$ is given by

$$
\mathcal{A}_{\text {tot }}=\mathcal{A}_{c} \otimes L\left(\mathcal{H}_{q}\right)=\mathbb{C}^{m} \otimes L\left(\mathcal{H}_{q}\right)=\oplus_{\alpha=0}^{m-1} L\left(\mathcal{H}_{q}\right),
$$

and it is convenient to realize $\mathcal{A}_{\text {tot }}$ as an algebra of operators on an auxiliary Hilbert space $\mathcal{H}_{\text {tot }}=\mathcal{H}_{q} \otimes \mathbb{C}^{m}=\oplus_{\alpha=0}^{m-1} \mathcal{H}_{q}$. $\mathcal{A}_{\text {tot }}$ is then isomorphic to the algebra of block diagonal $m \times m$ matrices $A=\operatorname{diag}\left(a_{0}, a_{1}, \ldots, a_{m-1}\right)$ with $a_{\alpha} \in L\left(\mathcal{H}_{q}\right)$. States on 
$\mathcal{A}_{\text {tot }}$ are represented by block diagonal matrices

$$
\rho=\operatorname{diag}\left(\rho_{0}, \rho_{1}, \ldots, \rho_{m-1}\right)
$$

where the $\rho_{\alpha}$ are positive trace class operators in $L\left(\mathcal{H}_{q}\right)$ with $\sum_{\alpha} \operatorname{Tr}\left(\rho_{\alpha}\right)=1$. By taking partial traces each state $\rho$ projects on a 'quantum state ' $\pi_{q}(\rho)$ and a 'classical state' $\pi_{c}(\rho)$ given respectively by

$$
\begin{gathered}
\pi_{q}(\rho)=\sum_{\alpha} \rho_{\alpha}, \\
\pi_{c}(\rho)=\left(\operatorname{Tr} \rho_{0}, \operatorname{Tr} \rho_{1}, \ldots, \operatorname{Tr} \rho_{m-1}\right) .
\end{gathered}
$$

The time evolution of the total system is given by a semi group $\alpha^{t}=e^{t L}$ of completely positive maps of $\mathcal{A}_{\text {tot }}$ - preserving hermiticity, identity and positivity with $L$ of the form

$$
L(A)=i[H, A]+\sum_{i=1}^{n}\left(V_{i}^{\star} A V_{i}-\frac{1}{2}\left\{V_{i}^{\star} V_{i}, A\right\}\right) .
$$

The $V_{i}$ can be arbitrary linear operators in $L\left(\mathcal{H}_{\text {tot }}\right)$ such that $\sum V_{i}^{\star} V_{i} \in \mathcal{A}_{\text {tot }}$ and $\sum V_{i}^{\star} A V_{i} \in \mathcal{A}_{\text {tot }}$ whenever $A \in \mathcal{A}_{\text {tot }}, H$ is an arbitrary block-diagonal self adjoint operator $H=\operatorname{diag}\left(H_{\alpha}\right)$ in $\mathcal{H}_{\text {tot }}$ and $\{$,$\} denotes anticommutator i.e.$

$$
\{A, B\} \equiv A B+B A .
$$

In order to couple the given quantum observable $A=\sum_{i=1}^{n} \lambda_{i} e_{i}$ to the classical system, the $V_{i}$ are chosen as tensor products $V_{i}=\sqrt{\kappa} e_{i} \otimes \phi_{i}$, where $\phi_{i}$ act as transformations on classical (pure) states. Denoting by $\rho(t)=\alpha_{t}(\rho(0))$, the time evolution of the states is given by the Liouville equation

$$
\dot{\rho}(t)=-i[H, \rho(t)]+\sum_{i=1}^{n}\left(V_{i} \rho(t) V_{i}^{\star}-\frac{1}{2}\left\{V_{i}^{\star} V_{i}, \rho(t)\right\}\right),
$$

where in general $H$ and the $V_{i}$ can explicitly depend on time.

A nice and simple example gives a model of a continuous measurement where a quantum spin $1 / 2$ system is coupled to a 2 -state classical system. In this situation we consider only one orthogonal projector $e$ on the two-dimensional Hilbert space $\mathcal{H}_{q}=\mathbb{C}^{2}$. The number of classical states is also two. To define the dynamics we choose the coupling operator $V$ in the following way:

$$
V=\sqrt{\kappa}\left(\begin{array}{ll}
0, & e \\
e, & 0
\end{array}\right) .
$$

The Liouville equation (1) for the density matrix $\rho=\operatorname{diag}\left(\rho_{0}, \rho_{1}\right)$ of the total system reads now

$$
\begin{aligned}
& \dot{\rho}_{0}=-i\left[H, \rho_{0}\right]+\kappa\left(e \rho_{1} e-\frac{1}{2}\left\{e, \rho_{0}\right\}\right), \\
& \dot{\rho}_{1}=-i\left[H, \rho_{1}\right]+\kappa\left(e \rho_{0} e-\frac{1}{2}\left\{e, \rho_{1}\right\}\right) .
\end{aligned}
$$


For this particularly simple coupling the effective quantum state $\hat{\rho}=\pi_{q}(\rho)=\rho_{0}+\rho_{1}$ evolves independently of the state of the classical system. One can say that here we have only transport of information from the quantum system to the classical one. We have:

$$
\dot{\hat{\rho}}=-i[H, \hat{\rho}]+\kappa\left(e \hat{\rho} e-\frac{1}{2}\{e, \hat{\rho}\}\right) .
$$

For the discussion of the quantum Zeno effect we specialize:

$$
\begin{aligned}
H & =\frac{\omega}{2} \sigma_{3}, \\
e & =\frac{1}{2}\left(\sigma_{0}+\sigma_{1}\right),
\end{aligned}
$$

$\sigma_{\mu}$ being the Pauli matrices.

We start with the quantum system being initially in the eigenstate of $\sigma_{1}$, and repeatedly (with "frequency" $\kappa$ ) check if the system is still in this state, each "yes" causing a flip in the coupled classical device - which we can continuously observe.

The evolution equation for $\hat{\rho}$, with the initial condition $\hat{\rho}(t=0)=e$, can be easily solved with the result:

$$
\hat{\rho}(t)=\frac{1}{2}\left(\sigma_{0}+x(t) \sigma_{1}+y(t) \sigma_{2}\right)
$$

where $x(t), y(t)$ are given by

$$
\begin{aligned}
& x(t)=\exp \left(\frac{-\kappa t}{4}\right)\left(\cosh \left(\frac{\kappa_{\omega} t}{4}\right)+\frac{\kappa}{\kappa_{\omega}} \sinh \left(\frac{\kappa_{\omega} t}{4}\right)\right), \\
& y(t)=\frac{4 \omega}{\kappa_{\omega}} \exp \left(\frac{-\kappa t}{4}\right) \sinh \left(\frac{\kappa_{\omega} t}{4}\right),
\end{aligned}
$$

where $\kappa_{\omega}=\sqrt{\kappa^{2}-16 \omega^{2}}$. Figure 1 shows this evolution, during the time interval $(0,4 \pi / \omega)$ for several different values of the dimensionless characteristic coefficient

$$
\alpha=\frac{\kappa}{4 \omega} .
$$

For $\alpha>1$ oscillations are damped completely, and then the distance travelled by the quantum state during the interaction becomes inversely proportional to the square root of $\alpha$. The natural distance in the state space is the geodesic Bures-Uhlmann distance $d \frown$, which is the geodesic distance for the Riemannian metric - given in our case by $d s^{2}=g_{i j} d x^{i} d x^{j}$, with $g_{i j}(\mathbf{v})=\left(\delta_{i j}+v_{i} v_{j} /\left(1-\mathbf{v}^{2}\right)\right)$. - cf. Refs 10, 11]. For density matrices $v=\left(\sigma_{0}+\mathbf{v} \cdot \sigma\right) / 2$ and $w=\left(\sigma_{0}+\mathbf{w} \cdot \sigma\right) / 2$ have (cf. Ref [12])

$$
d(v \frown w)=\frac{1}{2} \arccos \left(\mathbf{v} \cdot \mathbf{w}+\sqrt[4]{1-\mathbf{v}^{2}} \sqrt[4]{1-\mathbf{w}^{2}}\right) .
$$


In particular, if one of the states, say $v$, is pure, then $\mathbf{v}^{2}=1$ and we obtain

$$
d(v \frown w)=\frac{1}{2} \arccos (\mathbf{v} \cdot \mathbf{w}) .
$$

For $v=\hat{\rho}(t), w=e=\left(\sigma_{0}+\sigma_{1}\right) / 2$, as in the Zeno model, we obtain

$$
d(\hat{\rho}(t) \frown e)=\frac{1}{2} \arccos (x(t)) .
$$

Notice that $e$, being a pure state, is on the boundary of the state space, and the $d_{-}$-distance from $e$ depends only on one of the two relevant coordinates $x, y$ - contrary to the Frobenius distance $\operatorname{Tr}\left((v-w)^{2}\right)$, which would involve both coordinates. Assuming now $\alpha \gg 1$ and $\kappa t \gg 1$, we get for $x(t), y(t)$ in (13) asymptotic formulae:

$$
\begin{aligned}
& x(t) \asymp 1-\frac{2 \omega^{2} t}{\kappa}+\ldots \\
& y(t) \asymp \frac{\omega}{2 \kappa}+\ldots
\end{aligned}
$$

Thus the distance reached by state is in this asymptotic region given by

$$
d \asymp \omega \sqrt{\frac{t}{\kappa}}
$$

The Liouville equation (7) describes time evolution of statistical states of the total system. The information it contains need not be a maximal available one. It can be shown that with the equation (7) there is naturally associated a piecewise deterministic Markov process (cf. Ref. [14, 15]) on the set of pure states of the total system. Knowing this process one can answer all kinds of questions about time correlations of the events, and also simulate the random behavior of the classical system coupled to a quantum one. We refer to Ref. [13] for the full story, here we will describe the particular case of interest.

Let $T_{t}$ be a one-parameter semigroup of (non-linear) transformations of rays in $\mathbb{C}^{2}$ given by

$$
T(t) \phi=\frac{\phi(t)}{\|\phi(t)\|},
$$

where

$$
\phi(t)=\exp \left(-i H t-\frac{\kappa}{2} e t\right) \phi .
$$

Suppose we start with the quantum system in a pure state $\phi_{0}$, and the classical system in a state $s_{0}$. Then $\phi_{0}$ starts to evolve according to the deterministic (but non-Schrödinger) evolution $T(t) \phi_{0}$ until a jump oocurs at time $t_{1}$. The time $t_{1}$ of the jump is governed by an inhomogeneous Poisson process with the rate function $\lambda(t)=\kappa\left\|e T(t) \phi_{0}\right\|^{2}$. Classical system switches from $s_{0}$ to $s_{1}$, while $T\left(t_{1}\right) \phi_{0}$ jumps 
to $\phi_{1}=e T\left(t_{1}\right) \phi_{0}$, and the process starts again. With the initial state being an eigenstate of $e, e \phi_{0}=\phi_{0}$, as in our Zeno model, and for large values of the coupling constant, the rate function $\lambda$ is approximately constant and equal to $\kappa$. Thus $1 / \kappa$ can be interpreted as the expected time interval between the succesive jumps. Strong coupling between the two systems, necessary for a manifestation of the Zeno effect, manifests itself with a high frequency of jumps.

\section{Concluding Remarks}

We have shown that the mechanism leading to the quantum Zeno effect can be analysed within a model interaction between a classical and a quantum system. Zeno's effect appears when a classical device, that is able to high frequency switching between two alternate states, is strongly coupled to a quantum system prepared in an appropriate initial state. The Hamiltonian evolution of the quantum system is then slowed down, and it stops completely in the limit of infinite coupling constant. The dynamical origin of the phenomenon is easily understood within our framework: the quantum system is prepared in a initial state that is on the attractor consisting of stationary states of the dissipative part of the Liouvillian. The phenomenon is thus characteristic not only of the particular model we have considered, but of the whole class of similar models.

The minimal piecewise deterministic random process that we have given can be used for computing time characteristic of the interaction, and also for numerical simulations of the phenomenon. It also shows that measuring of the jump frequency of the classical apparatus can be used for an effective estimation of the value of the coupling constant.

Acknowledgments: The support of Alexander von Humboldt Foundation is acknowledged with thanks.

\section{References}

[1] B. Misra and E.C.G Sudarshan, The Zeno's paradox in quantum theory, J. Math. Phys., 18 (1977), 756-763

[2] L. Accardi, The probabilistic roots of the Quantum Mechanical Paradoxes, in The Wave-Particle Dualism, S.Diner (eds) 294-330, S. Reidel (1984)

[3] D. Home and M.A.B. Whitaker, A critical re-examination of the quantum Zeno paradox, J. Phys. A 25 (1992), 657-664

[4] E. Joos, Continuous measurement: Watchdog effect versus golden rule, Phys. Rev D 29 (1984), 1626-1633 
[5] H. Fearn and W.E. Lamb, Jr. Computational approach to the quantum Zeno effect. Position measurements. Phys. Rev. A 46 (1992), 1199-1205

[6] R.J. Cook, What are Quantum Jumps, Phys. Scr. T21 (1988) 49-51

[7] W.M. Itano, D.J. Heinzen, J.J. Bollinger, and D.J. Wineland, Quantum Zeno effect, Phys. rev. A 41 (1990), 2295-2300

[8] S. Pascazio, M. Namiki, G. Badurek and H. Rauch, Quantum Zeno effect with neutron spin, Phys. Lett. A 179 (1993) 155-160

[9] Ph. Blanchard and A. Jadczyk On the Interaction Between Classical and Quantum Systems, Phys. Lett. A 175 (1993), 157-164

[10] A. Jadczyk, On Berry's Phase, Paper presented at the Sth Summer Workshop on Mathematical Physics - Group Theoretical Methods and Physical Applications, Clausthal, July 1988

[11] A. Uhlmann, The Metric of Bures and the Geometric Phase, NTZ-Preprint-Nr $1 / 1992$

[12] M. Hübner, Explicit computation of the Bures distance for density matrices, Preprint-Nr.21/1991, Naturwiss. Theoret. Zentrum, Universität Leipzig

[13] Ph. Blanchard and A: Jadczyk, On Random Processes Associated with QuantoClassical Interactions. in preparation

[14] M.H.A. Davis, Lectures on Stochastic Control and Nonlinear Filtering, Tata Institute of Fundamental Research, Bombay 1984

[15] O.L. Costa and M.H.A. Davis, Approximation for Optimal Stopping of a Piecewise-Deterministic Process, Math. Control Signals Systems 1 (1988), 123146 


\section{Figure Captions}

Figure 1: Path travelled by the quantum state during the time interval $\{0,4 \pi / \omega\}$, for $\alpha=10^{-1}, 10^{0}, 10^{1}, 10^{2}$ respectively. For large values of $\alpha$ quantum Zeno effect reveals itself: the distance travelled by the state is $\asymp \sqrt{\frac{\pi}{\alpha}}$ 